\title{
COMPUTATION ON THE CAPACITY OF COMPOUND CHANNEL
}

\section{LEI LIN}

School of Mathematics and Systems Science

Guangdong Polytechnic Normal University

Guangzhou 510665

P. R. China

e-mail: linlei2@mail2.sysu.edu.cn

\begin{abstract}
We present an algorithm to compute the capacity of compound channel, which is specified by maximin of mutual informations. The algorithm utilizes the alternate optimization method similar to Arimoto-Blahut algorithm except for the more complex optimization method at the second phase. By transforming the original problem with non-differentiable objective function into a convex optimization problem with differentiable objective function, we obtain the necessary and sufficient conditions for the capacity-achieving input distributions. We also derive both upper and lower bounds of the maximin value, which provide a criterion to terminate any iterative methods. In addition, the convergence of the algorithm is proved. Finally, the numerical results are presented.
\end{abstract}

\section{Introduction}

In the early 1948, Shannon [1] introduced an important concept in information theory, channel capacity, which is to specify the asymptotic limit on the maximum rate at which information can be transmitted 2010 Mathematics Subject Classification: 68P30, 94A40, $94 \mathrm{~A} 15$.

Keywords and phrases: Arimoto-Blahut algorithm, convex optimization, compound channel, maximin of mutual informations.

Received June 10, 2017

(C) 2017 Scientific Advances Publishers 
reliably over a channel. For the discrete memoryless channel with single input and single output, the capacity can be efficiently computed by the Arimoto-Blahut algorithm, which is an alternate optimization algorithm ([2], [3]).

In this paper, we focus on the computation of the capacity of discrete memoryless compound channel with $m$ inputs and $n$ outputs ([4], [5]), which can be specified by a collection of probability transition matrices $\left\{Q_{k}(j \mid i), 1 \leq i \leq m, 1 \leq j \leq n\right\}_{1 \leq k \leq K}$. The compound channel can also be considered as a special finite state channel with the channel state unknown initially and kept unchanged during the transmission. As is well known, the capacity of a compound channel is given by

$$
C=\max _{p \in \mathbf{p}^{m}} \min _{1 \leq k \leq K}\left\{I\left(X ; Y_{k}\right)\right\},
$$

where $I\left(X ; Y_{k}\right)$ is the mutual information between the input and the output corresponding to the $k$-th channel probability transition matrix $\left[Q_{k}(j \mid i)\right]$ and

$$
\mathbf{p}^{m}=\left\{p \in \mathbf{R}^{m} \mid p(i) \geq 0, \sum_{i} p(i)=1\right\}
$$

is the set of all probability distributions on the channel input. Here we use $X$ without the subscript $k$ to indicate that the sender must design an encoder that is robust for all possible $k$, while we use $Y_{k}$ to indicate that the receiver knows the channel law exactly. The maximin in (1) is also the maximum information rate at which common messages can be broadcasted reliably from one sender to multiple receivers [6]. However, note that the compound channel capacity is, in general, not equal to the minimum of the capacities of the different channels in the family, as the capacity-achieving input distributions may be different for different channels in the family.

The paper is organized as follows. Firstly, the analytical properties of the minimum mutual informations such as continuity, concavity and differentiability are analyzed. The necessary and sufficient conditions for 
the capacity-achieving input distributions are revealed. Both lower bounds and upper bounds are derived, which provide a criterion to terminate any iterative algorithms when the capacity has been approximated sufficiently closely. Secondly, an iterative method to compute the maximin value of $K$ mutual informations is derived. Thirdly, the convergence of the proposed algorithm is proved. Finally, some numerical results are presented.

\section{Necessary and Sufficient Conditions}

Consider the compound channel described above. Let $Q_{k}=\left[Q_{k}(j \mid i)\right]$ be the $k$-th probability transition matrix. For any given distribution $p \in \mathbf{p}^{m}$, define

$$
f(p)=\min _{1 \leq k \leq K}\left\{I\left(p, Q_{k}\right)\right\}
$$

where

$$
I\left(p, Q_{k}\right) \triangleq I\left(X ; Y_{k}\right)=\sum_{i, j} p(i) Q_{k}(j \mid i) \log \frac{Q_{k}(j \mid i)}{q_{k}(j)}
$$

and

$$
q_{k}(j)=\sum_{i} p(i) Q_{k}(j \mid i) \quad \text { for } \quad 1 \leq k \leq K
$$

Proposition 1. The function $f(p)$ is continuous and concave over $\mathbf{p}^{m}$.

Proof. The continuity is obvious, while the concavity follows from the concavity of $I\left(p, Q_{k}\right)$ and the fact that minimum operation preserves concavity ([7], p. 80).

Hence, there exists $p^{*}$ such that $f\left(p^{*}\right)=\max _{p \in \mathbf{p}^{m}} f(p)$. However, since $f(p)$ may be non-differentiable, the Karush-Kuhn-Tucker (KKT) conditions may not exist. Fortunately, we can verify that maximizing $f(p)$ is equivalent to 


$$
\begin{gathered}
\max _{p, r}\{r\} \\
\text { s.t. } \begin{cases}r & \leq I\left(p, Q_{k}\right) \text { for } 1 \leq k \leq K ; \\
\sum_{i} p(i) & =1 ; \\
p(i) & \geq 0,\end{cases}
\end{gathered}
$$

which is a convex optimization problem since $r-I\left(p, Q_{k}\right)$ for $1 \leq k \leq K$ are convex in $p$ [8] and $r$ is linear. By defining

$$
L(\lambda, \nu)=r+\lambda\left(\sum p(i)-1\right)+\sum_{k} \nu_{k}\left(I\left(p, Q_{k}\right)-r\right),
$$

where $\lambda, v_{k}$ are Lagrange multipliers, we have the following KKT conditions:

$$
\begin{aligned}
& \left.\frac{\partial L(\lambda, \nu)}{\partial p(i)}\right|_{p(i)=p^{*}(i)}=0 \text { for } p^{*}(i)>0 \\
& \left.\frac{\partial L(\lambda, \nu)}{\partial p(i)}\right|_{p(i)=p^{*}(i)} \leq 0 \text { for } p^{*}(i)=0 \\
& \left.\frac{\partial L(\lambda, \nu)}{\partial r}\right|_{r=r^{*}}=1-\sum_{k} \nu_{k}=0 \\
& \nu_{k}\left(I\left(p^{*}, Q_{k}\right)-r^{*}\right)=0 ; v_{k} \geq 0
\end{aligned}
$$

From (6), we obtain

$$
\begin{array}{ll}
\sum_{k} \nu_{k} T_{k}(i)_{p^{*}}=\log e-\lambda & \text { for } p^{*}(i)>0 ; \\
\sum_{k} \nu_{k} T_{k}(i)_{p^{*}} \leq \log e-\lambda & \text { for } p^{*}(i)=0,
\end{array}
$$

where

$$
T_{k}(i)_{p^{*}}=\sum_{j} Q_{k}(j \mid i) \log \frac{Q_{k}(j \mid i)}{\sum_{i} Q_{k}(j \mid i) p^{*}(i)}
$$

Multiplying both sides of (7) by $p^{*}(i)$ and summing over $i$, it can be seen that 


$$
\sum_{k} \nu_{k} I\left(p^{*}, Q_{k}\right)=\log e-\lambda
$$

Subtracting $r^{*}=\max _{p \in \mathbf{p}^{m}} f(p)$ from both sides of (9) and utilizing $\nu_{k}\left(I\left(p^{*}, Q_{k}\right)-r^{*}\right)=0$, we obtain

$$
r^{*}=\log e-\lambda,
$$

and Theorem 1.

Theorem 1. For an input probability vector $p^{*}$ satisfying that $C=f\left(p^{*}\right)=\max _{p \in \mathbf{p}^{m}} f(p)$, there exists a vector $v=\left(v_{1}, v_{2}, \ldots, v_{k}\right)\left(v_{k} \geq 0\right)$ satisfying $\sum_{k} \nu_{k}=1$ such that

$$
\begin{array}{ll}
\sum_{k} v_{k} T_{k}(i)_{p^{*}}=C & \text { for } p^{*}(i)>0 ; \\
\sum_{k} v_{k} T_{k}(i)_{p^{*}} \leq C & \text { for } p^{*}(i)=0 .
\end{array}
$$

Theorem 2. For any probability vector $p$ and nonnegative vector $\nu=\left(\nu_{1}, \nu_{2}, \ldots, \nu_{k}\right)\left(\nu_{k} \geq 0\right)$ satisfying $\sum_{k} v_{k}=1$, the following hold:

$$
I_{L}(p) \leq C \leq I_{U}(p)
$$

where

$$
\begin{aligned}
& I_{L}(p)=\min _{k}\left\{I\left(p, Q_{k}\right)\right\}, \\
& I_{U}(p)=\min _{\nu} \max _{1 \leq i \leq m}\left\{\sum_{k} \nu_{k} T_{k}(i)_{p}\right\} .
\end{aligned}
$$

Proof. The lower bound is obvious, while the proof of the upper bound is similar to Problem 4.17 in ([9], p. 524). Actually, for any $\nu\left(v_{k} \geq 0\right)$ satisfying $\sum_{k} \nu_{k}=1$, it can be seen that

$$
\max _{1 \leq i \leq m}\left\{\sum_{k} v_{k} T_{k}(i)_{p}\right\}
$$




$$
\begin{aligned}
& =\sum p^{*}(i) \max _{1 \leq i \leq m}\left\{\sum_{k} v_{k} T_{k}(i)_{p}\right\} \\
& \geq \sum p^{*}(i)\left(\sum_{k} v_{k} T_{k}(i)_{p}\right) \\
& =\sum_{k} \nu_{k}\left(\sum p^{*}(i) T_{k}(i)_{p}\right) \\
& \geq \sum_{k} v_{k}\left(\sum p^{*}(i) T_{k}(i)_{p^{*}}\right)=C .
\end{aligned}
$$

Remark. If $p^{*}$ achieve $C$, from Theorem 1 and Theorem 2 we get $I_{L}\left(p^{*}\right)=I_{U}\left(p^{*}\right)$.

\section{The Iterative Optimizing Algorithm}

The computation of $C$ will be derived from the following theorem:

Theorem 3. Suppose that the channel transition matrices $Q_{1}, \ldots, Q_{K}$ are $m \times n$. For any $n \times m$ transition matrices $B_{1}, \ldots, B_{K}$, let

$$
\begin{aligned}
F\left(p, Q_{k}, B_{k}\right) & =\sum_{i} \sum_{j} p(i) Q_{k}(j \mid i) \log \frac{B_{k}(i \mid j)}{p(i)}, \\
J(p, B) & =\min _{1 \leq k \leq K}\left\{F\left(p, Q_{k}, B_{k}\right)\right\} .
\end{aligned}
$$

Then the following is true:

(1)

$$
C=\max _{p} \max _{B_{1}, \ldots, B_{K}} J(p, B) .
$$

(2) For fixed $p, J(p, B)$ is maximized by

$$
B_{k}^{*}=\frac{p(i) Q_{k}(j \mid i)}{\sum_{i} p(i) Q_{k}(j \mid i)}, 1 \leq k \leq K .
$$

(3) For fixed $B_{1}, \ldots, B_{K}, J(p, B)$ is maximized by 


$$
p^{*}(i)=\frac{\exp \left(\sum_{k} \mu_{k} \sum_{j} Q_{k}(j \mid i) \log B_{k}(i \mid j)\right)}{\sum_{i} \exp \left(\sum_{k} \mu_{k} \sum_{j} Q_{k}(j \mid i) \log B_{k}(i \mid j)\right)},
$$

where

$$
\mu_{k} \geq 0, \sum_{k} \mu_{k}=1
$$

and $\mu=\left(\mu_{1}, \ldots, \mu_{K}\right)=e_{l}$ such that $p^{*}$ satisfies that $F_{l}\left(p^{*}\right) \leq F_{j}\left(p^{*}\right)$ for all $j \neq l$ or $\mu=\mu^{0}$ such that $F_{l}\left(p^{*}\right)=F_{m}\left(p^{*}\right)$ for all $\mu_{l}^{0}, \mu_{m}^{0} \neq 0$ and $F_{l}\left(p^{*}\right) \leq F_{j}\left(p^{*}\right)$ for all $\mu_{j}^{0}=0$. Hereafter, for fixed $B_{k}$, we use $F_{k}(p) \triangleq F\left(p, Q_{k}, B_{k}\right)$ for convenience and $e_{l}$ states $K$-dimensional unit vector, that is, the l-th component of $e_{l}$ is 1 and the rest is 0 .

Proof. (1) From Theorem 1 in [3], it can be seen that $F\left(p, Q_{k}, B_{k}\right)$ $\leq F\left(p, Q_{k}, B_{k}^{*}\right)$ for fixed $p$. So it is obvious that

$$
J(p, B) \leq J\left(p, B^{*}\right),
$$

which means that $f(p)=\max _{B_{1}, \ldots, B_{K}} J(p, B)$.

(2) This fact is an immediate consequence of (19).

(3) Since $J(p, B)$ may be non-differentiable over $p$, Lagrange multiplier method can not be used. Fortunately, we can still transform the problem of maximizing $J(p, B)$ into an equivalent optimization problem

$$
\begin{gathered}
\max _{p, r}\{r\} \\
\text { s.t. }\left\{\begin{aligned}
r-F\left(p, Q_{k}, B_{k}\right) \leq 0 & \text { for } 1 \leq k \leq K, \\
\sum_{i} p(i)-1 & =0 .
\end{aligned}\right.
\end{gathered}
$$


Here, we ignore the constraint $p(i) \geq 0$ temporarily. Similar to Problem (5), it can be verified that Problem (20) is a convex optimization problem. By defining

$$
L(\lambda, \mu)=r+\lambda\left(\sum p(i)-1\right)+\sum \mu_{k}\left(F_{k}(p)-r\right),
$$

the following KKT conditions:

$$
\begin{aligned}
\left.\frac{\partial L(\lambda, \mu)}{\partial r}\right|_{r=r^{*}} & =0 ; \\
\left.\frac{\partial L(\lambda, \mu)}{\partial p(i)}\right|_{p(i)=p^{*}(i)} & =0 ; \\
\mu_{k}\left(F_{k}\left(p^{*}\right)-r^{*}\right) & =0 \text { for } 1 \leq k \leq K ; \\
\mu_{k} & \geq 0 \text { for } 1 \leq k \leq K,
\end{aligned}
$$

are true. The Equation (18) can be easily derived from the second constraint of (21) and the condition $\sum_{i} p^{*}(i)=1$. Solving from the first equation of the above KKT conditions, we get $\sum_{k} \mu_{k}=1$. In addition, for $\mu$, there are two kinds of cases. The one is $\mu=e_{l}$, that is, $\mu_{l}=1$ and $\mu_{j}=0$ for all $j \neq l$. From the condition $\mu_{l}\left(F_{l}\left(p^{*}\right)-r^{*}\right)=0$, it is obvious that $r^{*}=F_{l}\left(p^{*}\right)$. Hence $p^{*}$ should satisfy $r^{*}=F_{l}\left(p^{*}\right) \leq F_{j}\left(p^{*}\right)$ for all $j \neq l$. The other one is $\mu=\mu^{0}$. For all $\mu_{l}^{0}, \mu_{m}^{0} \neq 0$ it is obvious that $F_{l}\left(p^{*}\right)=r^{*}$ and $F_{m}\left(p^{*}\right)=r^{*}$, which means that $F_{l}\left(p^{*}\right)=F_{m}\left(p^{*}\right)$. Meanwhile, for all $\mu_{j}^{0}=0, F_{l}\left(p^{*}\right)=r^{*} \leq F_{j}\left(p^{*}\right)$ should be satisfied. Moreover, substituting (18) into (16) it can be seen that

$$
r^{*}=\log \sum_{i} \exp (c(i))
$$

Notice that in any case $p^{*}$ is always positive so that the constraint $p^{*}(i) \geq 0$ is not operative. 
Algorithm 1. Maximin of Mutual Information Algorithm

(1) Initialization: Choose an arbitrary probability vector $p(p(i)>0)$ and a very small number $\varepsilon$.

(2) Iteration:

Step 2.1. Compute $T_{k}(i)_{p}$ and $I\left(p, Q_{k}\right)$ for $1 \leq k \leq K$; if $I_{U}(p)-$ $I_{L}(p)<\varepsilon$, output $C=I_{L}(p)$ and exit the iterations; otherwise, compute $B_{k}^{*}$ for $1 \leq k \leq K$ according to (17).

Step 2.2. Fix $B_{k}=B_{k}^{*}$ and find $p^{*}$ according to (18) by identifying the true solution of $\mu$ :

Case 1. Let $\mu=e_{l}$ and compute $p^{*}$ and $F_{k}\left(p^{*}\right)$ for $1 \leq k \leq K$; if $F_{l}\left(p^{*}\right) \leq F_{j}\left(p^{*}\right)$ for all $j \neq l$, set $p=p^{*}$ and go to Step 2.1 .

Case 2. If Case 1 does not occur, search $\mu^{0}$ such that $p^{*}$ satisfies $F_{l}\left(p^{*}\right)=F_{m}\left(p^{*}\right)$ for all $\mu_{l}^{0}, \mu_{m}^{0} \neq 0$ and $F_{l}\left(p^{*}\right) \leq F_{j}\left(p^{*}\right)$ for all $\mu_{j}^{0}=0$. Set $p=p^{*}$ and go to Step 2.1.

Remark. In this algorithm, we utilize simplex method to compute $I_{U}(p)$ and Newton method to search $\mu^{0}$.

\section{Proof of the Convergence of the Algorithm}

In this section, we use a method similar to that of [2] and [10] to prove the convergence of the algorithm. For simplicity, let

$$
\begin{aligned}
C(t, t) & =\max _{B_{1}, B_{2}} J\left(p^{t}, B\right), \\
C(t+1, t) & =\max _{p} J\left(p, B^{t}\right),
\end{aligned}
$$

where $p^{t}$ stands for the input probability vector in the $t$-th iteration and $B_{k}^{t}(i \mid j)=\frac{p^{t}(i) Q_{k}(j \mid i)}{\sum_{i} p^{t}(i) Q_{k}(j \mid i)}$. 
Theorem 4. Let $p^{1} \in \mathbf{p}^{m}$ with all components strictly positive. Then the values $C(t, t)$ converge monotonically from below to $C$ as $t \rightarrow \infty$.

Proof. According to the Algorithm 1, the following relations are obvious:

$$
C(t, t) \leq C(t+1, t) \leq C(t+1, t+1) \leq \ldots \leq C .
$$

From (22), it is obvious that $C(t+1, t)=\log \sum_{i} c^{t}(i)$, where

$$
c^{t}(i)=\exp \left(\sum_{k} \mu_{k} \sum_{j} Q_{k}(j \mid i) \log B_{k}^{t}(i \mid j)\right)
$$

Now suppose that $p^{*}$ is an optimal probability vector, then

$$
\begin{aligned}
& \sum_{i} p^{*}(i) \log \frac{p^{t+1}(i)}{p^{t}(i)} \\
& \quad=\sum_{i} p^{*}(i) \log \left(\frac{1}{p^{t}(i)} \cdot \frac{c^{t}(i)}{\sum_{i} c^{t}(i)}\right) \\
& \quad=-C(t+1, t)+\sum_{k} \mu_{k} \sum_{i, j} p^{*}(i) Q_{k}(j \mid i) \log \frac{Q_{k}(j \mid i)}{q_{k}^{t}(j)} \\
& \quad=-C(t+1, t)+\sum_{k} \mu_{k} \sum_{i, j} p^{*}(i) Q_{k}(j \mid i)\left(\log \frac{Q_{k}(j \mid i)}{q_{k}^{*}(j)}+\log \frac{q_{k}^{*}(j)}{q_{k}^{t}(j)}\right) \\
& \quad \stackrel{(a)}{\geq} C-C(t+1, t)+\sum_{k} \mu_{k} \sum_{j} q_{k}^{*}(j) \log \frac{q_{k}^{*}(j)}{q_{k}^{t}(j)},
\end{aligned}
$$

where ( $a$ ) follows from $C \leq I\left(p^{*}, Q_{k}\right)$ for all $1 \leq k \leq K$ and $\sum_{k} \mu_{k}=1$. Because the last term of (25) are nonnegative (both $\mu_{k}$ and the relative entropies are nonnegative), for any $t$,

$$
C-C(t+1, t) \leq \sum_{i} p^{*}(i) \log \left(p^{t+1}(i) / p^{t}(i)\right),
$$

is true. Hence, summing up (26) over $t$ from 1 to $N$, 


$$
\begin{aligned}
\sum_{t=1}^{N}[C-C(t+1, t)] & \leq \sum_{i} p^{*}(i) \log \left(p^{N}(i) / p^{1}(i)\right) \\
& \leq \sum_{i} p^{*}(i) \log \left(p^{*}(i) / p^{1}(i)\right),
\end{aligned}
$$

for any integer $N \geq 1$. Note that $\sum_{i} p^{*}(i) \log \left(p^{*}(i) / p^{1}(i)\right)$ is a finite constant. Now assume that the limit of $C(t+1, t)$ is not equal to $C$. Since $C-C(t+1, t)$ is nonnegative and non-increasing with increasing $t$, the left hand of (27) would go to infinity, which is a contradiction. Therefore, we have

$$
\lim _{t \rightarrow \infty} C(t+1, t)=\lim _{t \rightarrow \infty} C(t, t)=C .
$$

\section{Numerical Results}

In this section, we present some examples in the cases of $K=2$ and $K=3$.

Example 1. Consider four pairs of channels with respective probability transition matrices as below:

A.

$$
Q_{1}=\left(\begin{array}{ccc}
0.53 & 0.07 & 0.40 \\
0.41 & 0.36 & 0.23 \\
0.45 & 0.50 & 0.05
\end{array}\right), Q_{2}=\left(\begin{array}{ccc}
0.37 & 0.59 & 0.04 \\
0.20 & 0.12 & 0.68 \\
0.37 & 0.32 & 0.31
\end{array}\right)
$$

B.

$$
Q_{1}=\left(\begin{array}{ccc}
0.18 & 0.31 & 0.51 \\
0.45 & 0.24 & 0.31 \\
0.65 & 0.20 & 0.15
\end{array}\right), Q_{2}=\left(\begin{array}{ccc}
0.13 & 0.48 & 0.39 \\
0.21 & 0.50 & 0.29 \\
0.15 & 0.08 & 0.77
\end{array}\right)
$$


C.

$$
Q_{1}=\left(\begin{array}{lll}
0.28 & 0.48 & 0.24 \\
0.01 & 0.43 & 0.56 \\
0.27 & 0.39 & 0.34
\end{array}\right), Q_{2}=\left(\begin{array}{ccc}
0.09 & 0.24 & 0.67 \\
0.51 & 0.05 & 0.44 \\
0.34 & 0.33 & 0.33
\end{array}\right), Q_{3}=\left(\begin{array}{lll}
0.09 & 0.40 & 0.51 \\
0.34 & 0.55 & 0.11 \\
0.51 & 0.43 & 0.06
\end{array}\right)
$$

D.

$$
Q_{1}=\left(\begin{array}{lll}
0.04 & 0.42 & 0.54 \\
0.35 & 0.38 & 0.27 \\
0.21 & 0.68 & 0.11
\end{array}\right), Q_{2}=\left(\begin{array}{ccc}
0.37 & 0.08 & 0.55 \\
0.05 & 0.50 & 0.45 \\
0.35 & 0.10 & 0.55
\end{array}\right), Q_{3}=\left(\begin{array}{lll}
0.46 & 0.07 & 0.47 \\
0.35 & 0.43 & 0.22 \\
0.48 & 0.17 & 0.35
\end{array}\right) \text {. }
$$

In our computation, we set $\varepsilon=10^{-6}$. The convergence of the algorithm can be seen from Figure 1 and Figure 2, in which $C_{A}, C_{B}, C_{C}$, and $C_{D}$ stand for the maximin values and $I_{L}(*), I_{U}(*)$ denote the lower and upper bounds of $*$, respectively.

\section{Conclusion}

In this paper, we have analyzed the analytical properties of the minimum mutual informations such as continuity, concavity, and differentiability. The necessary and sufficient conditions for the capacityachieving input distributions have been characterized. Both lower and upper bounds are derived, which provide a criterion to terminate any iterative algorithms when the capacity has been approximated sufficiently closely. Based on these, an iterative method to compute the maximin value of $K$ mutual informations has been derived. Finally, the convergence of the algorithm was proved. 


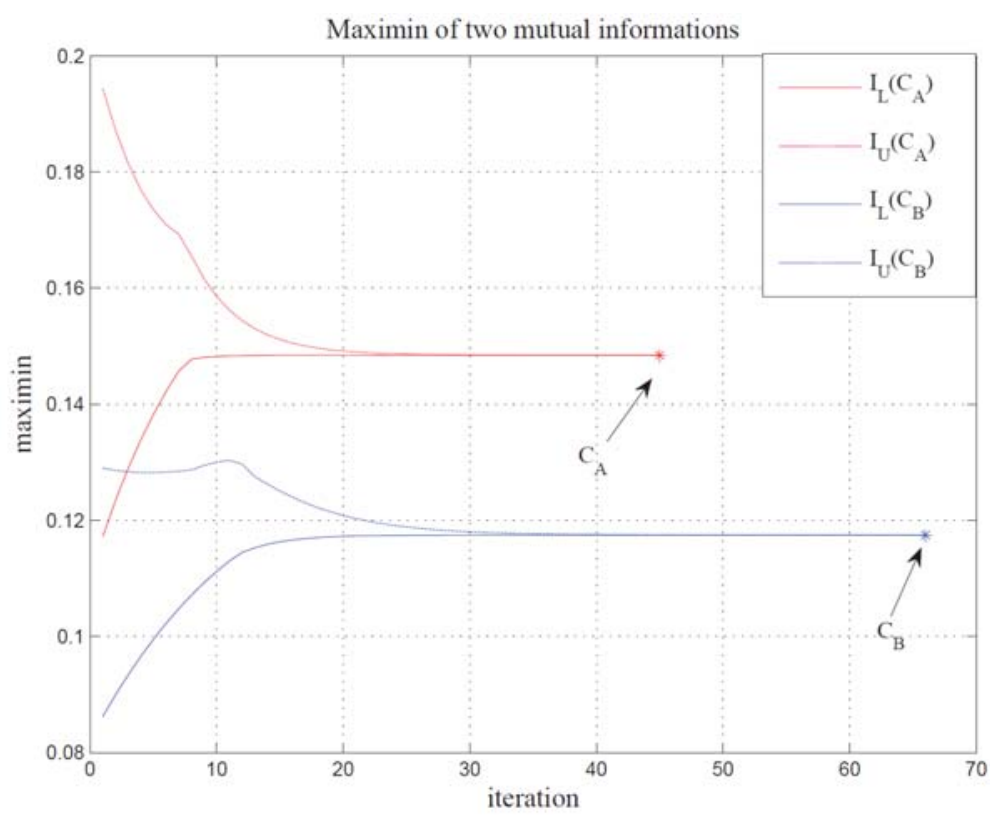

Figure 1. In the case of $K=2$.

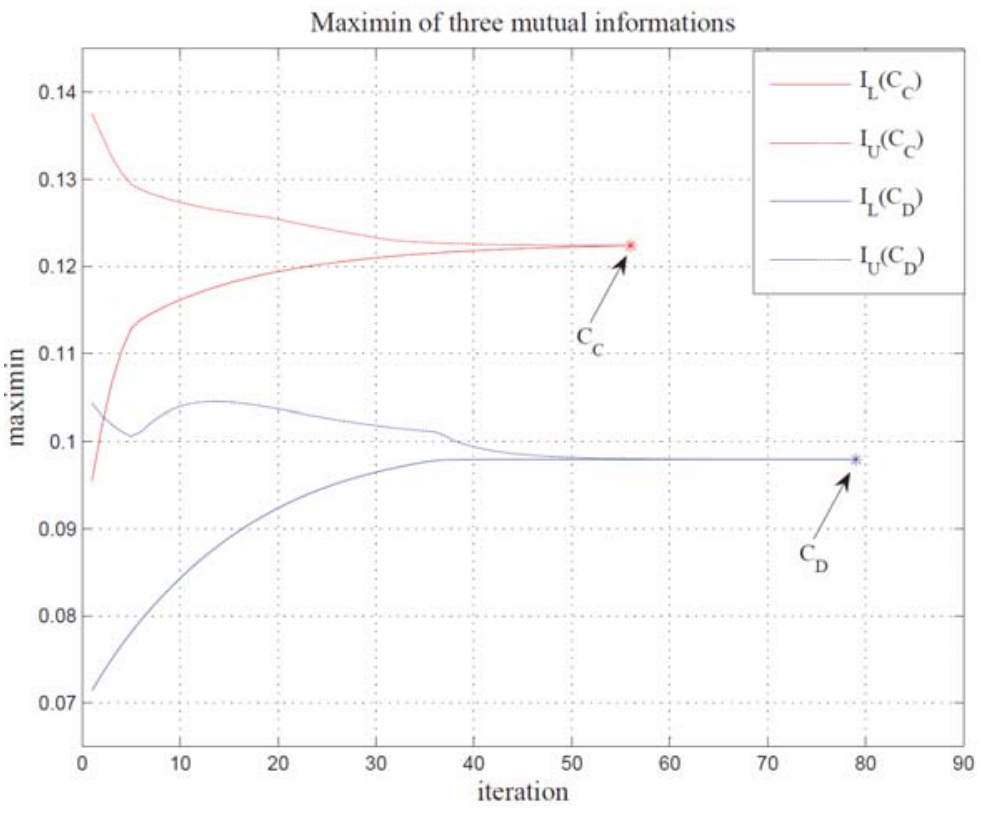

Figure 2. In the case of $K=3$. 


\section{References}

[1] C. E. Shannon, A mathematical theory of communication, Bell Syst. Tech. J. 27 (1948), 379-426; 623-656.

[2] S. Arimoto, An algorithm for computing the capacity of arbitrary discrete memoryless channels, IEEE Trans. Inform. Theory IT-18(1) (1972), 14-20.

[3] R. E. Blahut, Computation of channel capacity and rate distortion functions, IEEE Trans. Inform. Theory IT-18(4) (1972), 460-473.

[4] D. Blackwell, L. Breiman and A. J. Thomasian, The capacity of a class of channels, Ann. Math. Statist. 30(4) (1959), 1229-1241.

[5] J. Wolfowitz, Coding Theorems of Information Theory, Springer-Verlag, Berlin, 1964.

[6] T. M. Cover, Broadcast channels, IEEE Trans. Inform. Theory IT-18(1) (1972), 2-14.

[7] S. Boyd and L. Vandenberghe, Convex Optimization, Cambridge University Press, Cambridge, 2004.

[8] T. M. Cover and J. A. Thomas, Elements of Information Theory, John Wiley and Sons, Inc., New York, 1991.

[9] R. G. Gallager, Information Theory and Reliable Communication, John Wiley and Sons, Inc., New York, 1968.

[10] I. Csiszár, On the computation for rate-distortion functions, IEEE Trans. Inform. Theory 20(1) (1974), 122-124. 